\title{
Potato Wound-Healing Tissues: A Rich Source of \\ Natural Antioxidant Molecules with Potential for Food Preservation
}

\author{
Keyvan Dastmalchi, Isabel Wang, and Ruth E. Stark*
}

${ }^{1}$ Department of Chemistry and Biochemistry, The City College of New York, City

University of New York Graduate Center Ph.D. Programs in Biochemistry and Chemistry, and CUNY Institute for Macromolecular Assemblies, New York, NY 10031, USA

*Corresponding Author:

Tel: +1 2126508916. E-mail: rstark@ ccny.cuny.edu 


\begin{abstract}
The need for safe, effective preservatives is a prominent issue in the food and drug industries, reflecting demand for natural alternatives to synthetic chemicals viewed as harmful to consumers and the environment. Thus, this study determined the identities and scavenging capacities of antioxidant metabolites produced as a response to potato tuber wounding, using activity-guided fractionation of polar extracts from a Yukon Gold cultivar that had previously exhibited exceptionally high radical-scavenging activity. Activity-guided fractionation using the $\mathrm{ABTS}^{\bullet+}$ radical scavenging assay and LC-MS with TOF-MS for compositional analysis of the most potent antioxidant fractions yielded identification of nine constituents: coumaroylputrescine; feruloylquinic acid; isoferuloylputrescine; ferulic acid; 22,25-dimethoxy-3-[[2,3,4-tri- $O$-methyl-6-O-(2,3,4,6tetra- $O$-methyl- $\beta$-D-glucopyranosyl)- $\beta$-D-glucopyranosyl]oxy]-(3ß)-lanost-9(11)-en-24-

one; 4-(2Z)-2-decen-1-yl-5-[1-(4-hydroxyphenyl)decyl]-1,2-benzenediol; $\quad 8$-[(2E)-3,7dimethyl-2,6-octadien-1-yl]-5-hydroxy-2,8-dimethyl-6-(3-methyl-2-buten-1-yl)-2H-1benzopyran-4,7(3H,8H)-dione; 3-[(2-O- $\beta$-D-glucopyranosyl- $\beta$-D-glucopyranosyl)oxy]20-[(6-O- $\beta$-D-xylopyranosyl- $\beta$-D-glucopyranosyl)oxy]-dammar-24-en-19-al; $\quad$ (3ß)-28oxo-28-(phenylmethoxy)oleanan-3-yl $\quad 2-O-\beta$-D-galactopyranosyl-3- $O$-(phenylmethyl)-, butyl ester $\beta$-D-glucopyranosiduronic acid. A positive correlation was observed between the scavenging activities and the polarities of the active fractions. The antioxidant capacities of the fractions were also characterised by monitoring the activity throughout a 45-minute assay period.
\end{abstract}

KEYWORDS: Solanum tuberosum; potato; LC-MS; TOF-MS; wound periderm; antioxidants; $\mathrm{ABTS}^{\circ+}$ scavenging activity 


\section{Introduction}

The development of safe, effective preservatives poses a significant challenge in the food and drug industries. The demand for sustainable, natural alternatives to synthetic preservatives, many of which are reported to have a negative impact on the environment as well as potentially carcinogenic effects on consumers, has been growing rapidly over the past decade (Nychas \& Tassou, 1999). Plant-based antioxidants have potential applications as natural preservatives, but their sources and potencies remain to be optimised. In particular, the potato (Solanum tuberosum) has been found to be a rich source of antioxidants with high capacities for free radical scavenging (Madiwale, Reddivari, Holm, \& Vanamala, 2011). In previous studies, the extracts from potato peels have also been reported to inhibit oxidative processes in food products (Kanatt, Chander, Radhakrishna, \& Sharma, 2005).

As the world's fourth largest crop after wheat, rice, and corn, the potato also ranks third in global consumption, demonstrating its importance as a staple in the human diet as well as a mainstay of the global economy (Al-Weshay \& Rao, 2012; Madiwale, et al., 2011; Thompson, et al., 2009). Wounding and mechanical damage of the potato tuber together with suboptimal healing conditions result in desiccation and allow for bacterial or fungal infection. These problems render as many as $40-50 \%$ of harvested potatoes unsuitable for human consumption and generate substantial waste by-products (Schieber \& Saldana, 2008). However, under proper conditions potatoes form a layer called the wound periderm to guard against pathogen invasion and dehydration, progressing through different stages of healing at successive time points: three days after wounding, the suberised closing layer is fully formed; by the seventh, development of the wound 
periderm is initiated (Dastmalchi, et al., 2014; Neubauer, Lulai, Thompson, Suttle, \& Bolton, 2012). Moreover, in conjunction with periderm formation, wounded potato tissues generate reactive oxygen species (ROS) such as superoxide, peroxide, and hydroxyl radicals, containing infection by triggering cell death and thus damaging the tuber tissue (Reyes, Villarrel, \& Cisneros-Zevallos, 2007).

It has been proposed that tubers produce antioxidant compounds to scavenge for ROS in response to such stress (Reyes, et al., 2007), displaying antioxidant activity (AOA) levels comparable to high-antioxidant herbs such as ginkgo and ginger (Kähkönen, et al., 1999). The reported phytochemicals are of significant nutritional and pharmacological interest: they can interfere with oxidative processes by disrupting chainbreaking reactions and scavenging for free radicals; and they are associated with antibacterial, antiallergic, and anticarcinogenic properties (Friedman, 1997; Madiwale, et al., 2011; Madiwale, Reddivari, Holm, \& Vanamala, 2012; Thompson, et al., 2009). Moreover, the periderm from potato tubers represents a major industrial by-product that offers a potentially rich source of natural antioxidants. To realistically assess their efficacy as preservatives, the antioxidants must be isolated and identified so that their activities in different food systems can be determined. Published reports of free radical scavenging activities typically utilise assays that are subject to shortcomings such as limited $\mathrm{pH}$ range, polarity, steric hindrance, and spectral interference (Magalhaes, Segundo, Reis, \& Lima, 2008). Therefore, we sought a versatile assay that could overcome these limitations. The resulting antioxidant activities then formed the basis for activity-guided fractionation and identification of the most potent extracts from the wound-healing potato tuber periderm tissues. 
The 2,2'-azinobis(3-ethylbenzothiazoline-6-sulfonic acid) ammonium salt (ABTS) decolorisation assay measures the ability of antioxidants to scavenge free radicals in potato wound periderm by means of a single electron transfer reaction. Its advantages stem from the solubility of $\mathrm{ABTS}^{-+}$in both hydrophilic and lipophilic systems and its ability to monitor AOA over a wide $\mathrm{pH}$ range as well as over time. The ABTS assay also offers improvements compared with the traditional 2,2-diphenyl-1-picrylhidrazyl (DPPH) assay reported previously (Reyes \& Cisneros-Zevallos, 2003; Reyes, et al., 2007), which can suffer from steric hindrance or spectral interference from other phytochemicals $(\mathrm{Re}$, et al., 1999).

A reverse-phase high-performance liquid chromatography (RP-HPLC) strategy using a non-linear gradient system was then employed for separation of phytochemical constituents present in the antioxidant-rich fractions of the wound tissue extract. Finally, liquid chromatography-mass spectrometry (LC-MS) and time-of-flight mass spectrometry (TOF-MS) made it possible to identify the chemical constituents present in the most active fractions. Using the techniques outlined above, this study aimed to isolate and identify the most important antioxidants produced during the wounding response of potato tubers by determining the AOA in Day-7 Yukon Gold polar extracts and the metabolites responsible for scavenging activity. This study builds upon the findings of Dastmalchi, et al. (2014), who found polar wound periderm extracts of the Yukon Gold cultivar at Day 7 to have the highest activity among four tubers with distinct russeting patterns, reflecting the strong antioxidant ability of a number of secondary metabolites (polyphenolic amines, flavonoid glycosides, and phenolic acids) involved in potato tissue wound healing. 
Specifically, we subjected the polar fractions of the Day-7 Yukon Gold extract obtained through RP-HPLC to the ABTS assay, in order to evaluate scavenging activity over a time period of 45 minutes, permitting detection of slow- as well as fast-acting antioxidants. To isolate the constituents present in the fractions obtained by HPLC, we used reverse-phase liquid chromatography-mass spectrometry (LC-MS), since the extract being fractionated is polar and its constituents are non-volatile. Hence, through the structural elucidation of highly active fractions and the characterisation of their bioactivities, we hoped to identify promising new sources of natural preservatives with dietary value. We also obtained new information about the phytochemical constituents produced during the potato tuber wound-healing process, uncovering several compounds that had not been identified previously in their native or wound periderms (Dastmalchi, et al., 2014; Dastmalchi, et al., 2015; Narvaez-Cuenca, Vincken, Zheng, \& Gruppen, 2012; Yang \& Bernards, 2007).

\section{Materials and Methods}

\subsection{Chemicals and reagents}

HPLC-MS grade acetonitrile, water, methanol (J. T. Baker, Phillipsburg, NJ), and formic acid (Sigma-Aldrich, St. Louis, MO) were used in HPLC, LC-MS, and TOF-MS analyses. 2,2'-Azinobis(3-ethylbenzothiazoline-6-sulfonic acid ammonium salt) (ABTS), 6hydroxy-2,5,7,8-tetramethylchromane-2-carboxylic acid (TCI, Tokyo, Japan) and potassium peroxosulfate (Sigma-Aldrich, St. Louis, MO) were used for the antioxidant assay. 


\subsection{Plant material}

Potato tuber cultivars of the Yukon Gold variety, 2011 crop year, were provided by Joe Nuñez, University of California Cooperative Extension (Davis, CA).

\subsection{Sample preparation}

Procedures for this work followed the methods described by Dastmalchi et al. (2014, 2015). Briefly, Yukon Gold potato tubers were peeled and sectioned longitudinally under sterile conditions using a mandolin slicer to obtain disks $\sim 5 \mathrm{~mm}$ in thickness. Disks were placed on wet cellulose filter paper and left for 7 days at $25{ }^{\circ} \mathrm{C}$ on wire netting supports within closed plastic boxes in a dark enclosure. Humidity was maintained by adding water to the bottom of the boxes. The newly formed brown surface layer of easily detached wound tissue was collected using a flat spatula. Harvested wound tissues were frozen immediately in liquid nitrogen and stored at $-80^{\circ} \mathrm{C}$ for further processing.

The wound tissues were extracted using a modification of the protocol employed by Choi, et al. (Choi, et al., 2004). A 10-mg portion of the freeze-dried material was extracted with $2 \mathrm{~mL}$ of $60 \%(\mathrm{v} / \mathrm{v})$ methanol-water by ultrasonication (Branson Ultrasonics, Danbury, CT) for $1 \mathrm{~min}$, followed by addition of $2 \mathrm{~mL}$ chloroform and sonication again for $1 \mathrm{~min}$. Each extract was then incubated at room temperature in a shaker for 10 min, followed by tabletop centrifugation (Beckman Coulter, Fullerton, CA) at $3000 \mathrm{rpm}$ to produce three separate phases: soluble polar, soluble nonpolar, and an interphase of suspended particulates. The upper soluble polar extracts were removed carefully with a glass Pasteur pipette and dried under a flow of nitrogen gas. 


\subsection{Fractionation of the extracts}

LC separation was performed using a $150 \times 4.6 \mathrm{~mm}, 3.0 \mu \mathrm{m}$ AscentisR C18 column (Supelco, Bellefonte, PA) operated by an Agilent 1200 Series HPLC liquid chromatograph equipped with a G1311A quaternary pump, G1322A degasser, G1316A temperature controller, and G1315B diode array detector coupled to a G1364C analytical fractionator (Agilent, Santa Clara, CA). Each analysis was performed by injecting a 30$\mu \mathrm{L}$ sample into the column and eluting with a flow rate of $0.4 \mathrm{~mL} / \mathrm{min}$. The mobile phase was composed of $0.1 \%$ aqueous formic acid (A) and $0.1 \%$ formic acid in acetonitrile (B). The following program of nonlinear gradient elution was used: $2 \% \mathbf{B}(0-5 \mathrm{~min}), 10 \% \mathbf{B}$ (5-8 min), $15 \%$ B (8-25 min), 100\% B (25-38 min), and 2\% B (38-50 min). Fractions were collected in time-based mode at 30-s intervals between 14 and 41 min during a 50min chromatographic run that was repeated twenty times to accumulate enough sample for concentration and analysis by LC-MS and TOF-MS.

\subsection{ABTS $^{*+}$ scavenging}

Antioxidant assessment of the polar extracts was conducted using the ABTS assay, following the method employed by Dastmalchi et al. (2014, 2015). The ABTS radical cation was produced by reacting a $140 \mathrm{mM}$ potassium persulfate $\left(\mathrm{K}_{2} \mathrm{~S}_{2} \mathrm{O}_{8}\right)$ oxidising agent with $7 \mathrm{mM}$ ABTS in the dark at ambient temperature for approximately 12 to 16 hours. Once sufficient ABTS was converted into ABTS, ${ }^{++}$resulting in decolorisation of the cation, its absorbance was adjusted to $0.70( \pm 0.02)$ at $734 \mathrm{~nm}$ by dilution with ethanol. A series of $2-\mu \mathrm{L}$ samples from each of the 30 periderm fractions was placed in individual wells of a 96-well microplate, with each fraction designated by a column of 8 replicates. 
The same amounts of ferulic acid and $60 \%$ methanol were added to columns designated as positive and negative controls, respectively; $198 \mu \mathrm{L}$ of $\mathrm{ABTS}^{\bullet+}$ were added to each well. Absorbance was recorded immediately after mixing using a Spectramax microplate reader (Molecular Devices, Sunnyvale, CA) set at $734 \mathrm{~nm}$, and at subsequent 5-min intervals for $45 \mathrm{~min}$. The percent inhibition values for each sample were then calculated using the equation:

$$
\left[\frac{\left(\text { Absorbance }_{\text {control }}-\text { Absorbance }_{\text {sample }}\right)}{\left(\text { Absorbance }_{\text {control }}\right)}\right] \times 100
$$

Inhibition values for 8 concentrations from 0.2 to $2.0 \mathrm{mM}$ of 6-hydroxy-2,5,7,8 tetramethylchromane-2-carboxylic acid (Trolox) were measured as a reference standard. The area underneath the curve (AUC) of the plot of percent inhibition versus reaction time was then calculated for each fraction. Calculating and comparing the AUC values assesses the levels of scavenging more accurately than the Trolox Equivalent Antioxidant Capacity (TEAC) value, which reflects only the capacity of the antioxidant to inhibit ABTS at a defined time point relative to Trolox. Moreover, the AUC usefully takes into account the rates of reaction of the various antioxidants with ABTS, thus reflecting total activity of both slow and fast-acting compounds.

The AUCs were determined using two different numerical methods. They were first calculated with SigmaPlot (Fig. 1, middle). The integration function of this program employs the trapezoidal rule (Whitaker \& Robinson, 1967), which computes the integral over $n$ subintervals of time using a linear piecewise interpolation between the data values obtained over each run. It uses the following equation: 


$$
\int_{a}^{b} f(x) d x \approx \sum_{i=1}^{n-1}\left[y\left(x_{i+1}-x_{i}\right)+\frac{1}{2}\left(y_{i+1}-y_{i}\right)\left(x_{i+1}-x_{i}\right)\right]
$$

These values were then crosschecked with Simpson's rule (Abramowitz \& Stegun, 1972), an alternative method of estimating definite integrals that approximates the function using quadratic instead of linear interpolation (Fig. 1, bottom) using the following equation:

$$
\int_{a}^{b} f(x) d x \approx \frac{\Delta \mathrm{x}}{3}\left[f\left(x_{0}\right)+4 f\left(x_{1}\right)+2 f\left(x_{2}\right)+\cdots+2 f\left(x_{n-2}\right)+4 f\left(x_{n-1}\right)+f\left(x_{n}\right)\right]
$$

\subsection{LC-MS}

Liquid chromatography was performed using a Shimadzu Ultra Fast Liquid Chromatograph equipped with two LC-20 AD pumps, an SIL-20 AC autosampler, and a CTO-20 AC column oven. Separation was carried out utilizing a $150 \times 4.6 \mathrm{~mm}$ Supelco AscentisR C18 column. The mobile phase was composed of solvents A, $0.1 \%$ aqueous formic acid and $\mathbf{B}, 0.1 \%$ formic acid in acetonitrile with a program of nonlinear elution: $2 \% \mathbf{B}(0-5 \mathrm{~min}), 2-10 \% \mathbf{B}(5-8 \mathrm{~min}), 10-15 \% \mathbf{B}(8-13 \mathrm{~min}), 15 \% \mathbf{B}$ (13-25 $\mathrm{min}), 15-$ $30 \%$ B (25-28 min), 30-40\% B (28-50 min), and 40-100\% B (50-60 min). The analysis was performed by injecting $10 \mu \mathrm{L}$ of each sample twice at a flow rate of $0.4 \mathrm{~mL} / \mathrm{min}$ at $35{ }^{\circ} \mathrm{C}$.

The LC system was coupled to an Applied Biosystems 4000Q Linear Ion Trap Quadrupole Trap (Q-Trap) mass spectrometer (Foster City, CA) in order to identify the chemical compounds present in each fraction through their $\mathrm{MS}^{2}$ fragmentations, using an unfractionated Yukon Day-7 extract as a reference standard. Spectra and full scan data were acquired in both positive and negative modes using electrospray ionisation (ESI), 
optimised to $66 \mathrm{~V}$ and $-140 \mathrm{~V}$, respectively, and with a source temperature of $300{ }^{\circ} \mathrm{C}$. Data collected over an $m / z$ range of 100-1300 were processed with Analyst 1.4.1 software; potato metabolites such as chlorogenic acid and rutin, which have been reported previously in potatoes (Dastmalchi, et al., 2014), were used to optimise the declustering potential.

\subsection{TOF-MS}

The fractions were infused at a rate of $0.01 \mathrm{~mL} / \mathrm{min}$ using a Harvard 11 Plus Syringe pump (Harvard Apparatus, Holliston, MA) into a Waters LCT XE TOF mass spectrometer (Micromass, Manchester, UK) in positive electrospray mode over the range of $m / z$ 100-1300. The software used for data analysis was MassLynx Version 4.1. The capillary voltage was maintained at $4000 \mathrm{~V}$ and the nitrogen gas flow was $300 \mathrm{~L} / \mathrm{h}$ for both desolvation and nebulisation. The desolvation and source temperatures were $150{ }^{\circ} \mathrm{C}$ and $80{ }^{\circ} \mathrm{C}$, respectively. The reference standard used for dynamic range enhancement was a solution of $200 \mathrm{pg} / \mathrm{mL}$ leucine/encephalin in 1:1 $\mathrm{v} / \mathrm{v}$ acetonitrile-water containing $0.1 \%$ formic acid. The reference was collected every five scans and data were collected using a scan time of $0.2 \mathrm{~s}$.

\subsection{Metabolite identification}

Based on the $m / z$ values of the molecular and fragment ions obtained from the TOF-MS experiments, elemental composition analysis was carried out using MassLynx V4.1 software. This procedure generated possible molecular formulas within the given margin of error. Using SciFinder Scholar, we considered all possible compounds with 
these formulas that had been isolated and identified previously in the Solanaceae plant family; their published molecular ions and fragmentation data were examined to find the molecular structure that best matched our experimental data.

\subsection{Statistical analysis}

The antioxidant results were presented as mean value \pm standard error. Analysis of variance was carried out using Tukey pairwise analysis procedures (Jackson, 2014). Correlations between data sets were calculated using Pearson's correlation values (Crawley, 2005). SigmaPlot version 13 was used for calculation of the areas under the curve. 


\section{Results and Discussion}

\subsection{Evaluation of ABTS $^{\bullet+}$ scavenging activity.}

The validity and accuracy of the ABTS assay for accurate evaluations of AOA in potato wound periderm were demonstrated using a positive control, ferulic acid in ethanol. The average of TEACs measured over all time points was $1.32 \mathrm{mM}$ Trolox/100 $\mathrm{g}$ (Table 1), confirming previously published results (El-Sayed \& Rabaliski, 2013). Among the 30 fractions that were collected, fractions 1-12 demonstrated significant percentage inhibition values at the end of the assay period (Fig. 1), whereas fractions 13-30 showed no antioxidant activity. Therefore the former fractions were examined further to elucidate their constituent phytochemical substances using LC-MS and MS-TOF analysis.

Since an RP-HPLC separation technique was employed, more polar compounds eluted first, producing well-resolved chromatograms (Supplementary Figure 1). Therefore one can deduce that the active fractions, 1-12, are the more polar ones and that there is a direct correlation between levels of scavenging activity and polarity. A similar trend was observed in previously studied plant systems for which the greatest antioxidant capacity was found in compounds with highest polarity; now we can extend this correlation to the wound periderm of potato (Bhandari \& Rastogi, 1983; Calliet, Lorenzo, Cote, Sylvain, \& Lacroix, 2013; Re, et al., 1999).

By comparing the respective percentage inhibitions at the end of the 45 -minute assay period as well as their cumulative antioxidant capacities (Fig. 1, top), we identified F4-F8 as the most active fractions. The trend in final percent inhibitions (Fig. 1, top) was confirmed by the AUC values calculated using two different numerical methods (Fig. 1, middle and bottom). Whereas the differences between the AUCs calculated by the two 
methods are negligible, as shown by a $99.9 \%$ correlation between the two sets of numbers, the values calculated with Simpson's Rule are expected to have a lower percentage error because this method uses a closer approximation to the function curve.

Ultimately, the percentage inhibition at 45 minutes and the AUC values over time were in excellent agreement: F4-F8 possessed both the highest AUC and average final percentage inhibition; likewise, the AUCs for the remaining fractions were directly proportional to their respective inhibition values. By using the AUC approach as well as comparing percent inhibitions, we were able to accurately characterise scavenging activity for both those antioxidants with lag phases (slow-acting) and those without delays (fast-acting). Likewise, the percentage-inhibition $v s$ time plots were able to provide additional information about the kinetics of the antioxidant constituents in each fraction (Fig. 1). Fractions F1-F4 demonstrated a largely linear time dependence of activities, which suggests the presence of slow-acting antioxidants (Figure 2). Conversely, the graphs for F5-F12, which generally show an initial rapid increase followed by a slower rise during the 20- to 45-minute assay period, imply that they likely contain predominantly fast-acting antioxidants (Fig. 1). Pearson correlation analyses (Crawley, 2005 ) of the data showed $>95 \%$ correlation of the AUC values with either the 0 -minute percentage inhibitions or the activities at $45 \mathrm{~min}$, indicating that the fractions contained both slow- and fast-acting antioxidants. This ability to categorise the rate of antioxidant action by monitoring the scavenging capacity during extended time periods offers further indication of the usefulness of protocols such as the ABTS ${ }^{\bullet+}$ scavenging assay. 
Among the most active fractions, we chose five of the most potent ones for further compositional analysis. The order of activity among these fractions based on the AUC values was F5 > F6 > F8 > F7 and F4.

\subsection{Metabolite identification}

The most active fractions, F4-F8, were further analysed by mass spectrometry using LC-MS Q-Trap and MS-TOF instruments; the chemical constituents present therein were then identified from spectra illustrated in Supplementary Figure 2 and are summarized in Table 2. The full scan data from the LC-MS Q-Trap analysis were compared with the ionic spectra from MS-TOF runs, in order to confirm the consistency of the two datasets. The active fraction F4 contained coumaroylputrescine (Table 2, compound 1), a compound with reported superoxide anion radical-scavenging activity (Narvaez-Cuenca, et al., 2012) that is thought to be an important part of the plant's nonenzymatic defence mechanism (Velikoca, Edreva, Tsonev, \& Jones, 2007). Ferulic acid (FA) was found in both F4 and F5 (Table 2, compound 2); this second compound has been reported previously for effective scavenging of superoxide anion radical and thus approved in several countries as a safe food additive to inhibit lipid peroxidation (Chung \& Champagne, 2011). Moreover, Compound 2 has also demonstrated significant levels of antifilarial activity against adult worms of $S$. cervi in comparison to those of antifilarial drugs in current use (Saini, Gayen, Nayak, \& Deepak, 2012). A third isolated component with $m / z 525.2873$ did not appear in prior published reports for this plant family.

Active fraction F5 was also found to contain feruloylquinic acid (FQA) (Table 2, Compound 3), using high-resolution mass spectrometry (TOF-MS) and by comparison with fragmentation data from prior studies (Wu, Meyer, Whitaker, Litt, \& Kennelly, 
2013). The presence of a catechol moiety and adjacent phenolic groups can explain the electron-donating ability and consequent free-radical-scavenging activity of this compound (Bendary, Francis, Ali, Sarwat, \& El-Hady, 2013). Compound 3 has been reported previously in our metabolomic investigation of polar extracts from wounded potato tissues as an abundant marker for the Norkotah Russet cultivar in both Day-3 and Day-7 extracts (Dastmalchi, et al., 2014). The compound has also been reported to have an inhibitory effect on enzymatic browning caused by polyphenol oxidase (PPOs) in mushroom and potato, proposed in turn to occur by inhibiting the production of $o$ quinone melanin precursors (Kuijpers, et al., 2014). This compound and its derivatives, which are also found in coffee, have exhibited diverse biological functions in animal models. For instance, they have the ability to suppress postprandial hyperglycaemia, hyperinsulinaemia and hyperlipidaemia through the inhibition of intestinal enzymes in mice (Murase, et al., 2012). A third component present with an $\mathrm{m} / z 349.1836$ failed to match prior reports for this plant family. However, the fragmentation pattern suggests that this compound is a ferulic acid derivative.

In fraction F6 we detected a molecular ion corresponding to the saponin 22,25dimethoxy-3-[[2,3,4-tri-O-methyl-6-O-(2,3,4,6-tetra-O-methyl- $\beta$-D-glucopyranosyl)- $\beta$ D-glucopyranosyl]oxy]-lanost-9(11)-en-24-one (Table 2, Compound 4), and its fragment ion corresponding to 26-(acetyloxy)-(24E)-lanosta-7,9(11),24-trien-3-one. This saponin has been isolated previously from the plant Trichosanthes palmate (Bhandari et al., 1983). The fragment aglycone was isolated from Ganoderma lucidum (Munehisa et al., 1986). Another compound present in F6 was the alkylphenol, Sarmentosumol F, designated as 4(2Z)-2-decen-1-yl-5-[1-(4-hydroxyphenyl)decyl]-1,2-benzenediol (Yang et al., 2013) 
(Table 2, Compound 5). As mentioned above, the presence of a catechol group and an adjacent phenolic group can explain the free-radical-scavenging ability of the compound and its possible contribution to the antioxidant activity of the extract. Another notable feature of this molecule is the presence of a long-chain hydrocarbon. This compound, isolated for the first time in the plant Piper sarmentosum, has been reported to possess antimicrobial activity against Escherichia coli, Staphylococcus aureus and Candida albicans microbial species. In addition, this compound demonstrated cytotoxicity against human myeloid leukaemia and in two human lung adenocarcinoma cell lines (Yang et al., 2013). All isolated components in fraction 6 were identified.

F7 was found to contain three other compounds of interest: isoferuloylputrescine, Compound $\quad 6, \quad 8$-[(2E)-3,7-dimethyl-2,6-octadien-1-yl]-5-hydroxy-2,8-dimethyl-6-(3methyl-2-buten-1-yl)-2H-1-benzopyran-4,7(3H,8H)-dione, Compound 7, and 3-[(2-O- $\beta$ D-glucopyranosyl- $\beta$-D-glucopyranosyl)oxy]-20-[(6-O- $\beta$-D-xylopyranosyl- $\beta$-Dglucopyranosyl)oxy]-dammar-24-en-19-al, Compound 8 (Table 2). Compound 6 itself has been reported to offer resistance to Phytophthora infestans infection in potatoes (Yogendra, et al., 2014). Compound 7 was first reported as a benzopryan derivative isolated from Mallotus apelta, a plant widely used to treat chronic hepatitis and showing moderate anti-microbial activity (An, Hu, Cheng, \& Chen, 2001). Moreover, its synthetic analogues have been found to exhibit substantial scavenging capacity as well as antiviral activity (Koufaki, et al., 2006). Compound 8, a steroidal saponin known as Gymnemaside $\mathrm{V}$, has been reported in Gymnema sylvestre, which is used in traditional medicine as a diuretic and cough remedy, as well in the treatment of diabetes (Yoshikawa, Arihara, Matsuura, \& Miyaset, 1992). This compound belongs to a class of phytochemical 
substances known as Gypenosides. These compounds have demonstrated bioactivities such as in vitro inhibition of pancreatic lipase which can be relevance in the treatment of hyperlipidaemia (Su, et al., 2016). These compounds are also being investigated for their effectiveness in cancer therapy since they induce p53-independent apoptosis in a lung carcinoma cell line (Liu, et al., 2015). The toxicological attributes of these constituents have not been studied previously; nevertheless, their reported activities suggest valuable potential as preservatives for the manufacture of food and cosmetics. All components of Fraction 7 were identified.

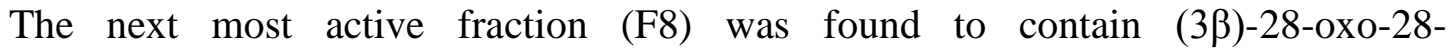
(phenylmethoxy)oleanan-3-yl 2-O- $\beta$-D-galactopyranosyl-3-O-(phenylmethyl)-, butyl ester of $\beta$-D-glucopyranosiduronic acid, (Compound 9, Table 2). This compound was first isolated from Panax japonicus, Japanese ginseng. Moreover, as a relatively scarce triperpenoid saponin in nature, its chemical synthesis has also been attempted, due to interest in its anti-fungal and ant-inflammatory properties (Liu, Fan, Li, Li, \& Guo, 2010). Similar derivatives have been reported to exhibit substantial DPPH-detected radical scavenging as well as melanogenesis-inhibitory activity (Zhang, et al., 2014). In addition, they have demonstrated anti-diabetic potential, whereby effective inhibition of the formation of advanced glycation end-products could suggest effectiveness against other mechanisms contributing to hyperglycaemia (Matsuda, Wang, Managi, \& Yoshikawa, 2003). Ultimately, although lower in scavenging capacity than Compound $\mathbf{3}$ found in F5, this identified saponin appears to display complementary bioactive functions. All components in Fraction 8 were identified successfully. 
Fractions 10 and 11 contained chlorogenic acid, which is a well-established antioxidant (Madiwale, et al., 2011; Nenadis, Wang, Tsimidou, \& Zhang, 2004) that is abundant in our wound tissue extracts (Dastmalchi, et al., 2014). Nonetheless the fractions containing chlorogenic acid were not among the most active fractions, possibly because of a potentiation effect for the constituents in the highly active fractions. Chlorogenic acid has been identified previously as a potential biomarker in the Yukon Gold Day-7 polar extract in a study of four potato cultivars with a gradient of russeting character (Dastmalchi, et al., 2014). This compound has been reported to have beneficial properties for human health, including antioxidant, hypoglycaemic, antiviral and hepatoprotective functions demonstrated in vitro, in vivo and in epidemiological studies (Farah \& Donangelo, 2006).

\section{Conclusions}

This study successfully identified the most active polar antioxidant metabolites produced in Yukon Gold potato periderm tissues 7 days after wounding. Moreover, by performing activity-guided fractionation on the wound periderm extract, we established a high degree of correlation between antioxidant capacity and polarity. This trend validates the important contributions of the most polar fractions to the scavenging capacity of the extract (Dastmalchi, et al., 2014; Dastmalchi, et al., 2015; Schieber, et al., 2008).

Many chemical constituents of the most potent antioxidant fractions were provisionally identified using LC-MS/MS and MS-TOF methods; structural elucidation of the two newly reported compounds in active fractions 4 and 5 should be possible with additional NMR measurements on larger samples. A useful follow-up study would 
involve screening of these fractions against potato pathogens to investigate whether the metabolites can offer protection to the potato tuber on a larger agricultural scale. Future studies could also evaluate interactions, such as synergism, potentiation, and antagonism between the phytochemical constituents, especially those involving the multiple active metabolites present in each of fractions 6, 7. Because compounds such as Sarmentosumol F, 8-[(2E)-3,7-dimethyl-2,6-octadien-1-yl]-5-hydroxy-2,8-dimethyl-6-(3-methyl-2-buten1-yl)-2H-1-benzopyran demonstrated significant bioactivities in various disease models, they could have potential as templates for drug development. Thus the findings of the current study provide motivation to further investigate the identified metabolites as natural preservatives with possible dietary value (Maqsood, Benjakul, \& Shahidi, 2013). Taking such an approach, it is possible to use antioxidant capacity assessment of wounded plant tissues to point the way toward phytochemicals that can be beneficial to the food industry.

\section{Acknowledgements}

Financial support for this work was obtained from the U.S. National Science Foundation (MCB-0843627, 1411984 and 0741914, CHEM-0520963). Infrastructural assistance was

provided by a grant from the National Institute on Minority Health and Health Disparities (8G12MD007603-29). Joe Nuñez (University of California Cooperative Extension) supplied the potato tubers for analysis. Professor David Jeruzalmi provided generous access to the microplate reader. Dr. Lijia Yang provided expert technical assistance and access to the LC/MS/MS and LC-TOF instruments. 


\section{References}

Abramowitz, M., \& Stegun, I. (1972). Handbook of mathematical functions with formulas, graphs, and mathematical tables: Dover.

Al-Weshay, A., \& Rao, V. A. (2012). Potato peel as a source of important phytochemical antioxidant nutraceuticals and their role in human health - A Review, phytochemicals as nutraceuticals - global approaches to their role in nutrition and health.

An, T.-Y., Hu, L.-H., Cheng, X.-F., \& Chen, Z.-L. (2001). Benzopyran derivatives from Mallotus apelta. Phytochemistry, 57, 273-278.

Bendary, E., Francis, R. R., Ali, H. M. G., Sarwat, M. I., \& El-Hady, S. (2013). Antioxidant and structure activity relationships (SARs) of some phenolic and anilin compounds. Annals of Agricultural Sciences, 58, 173-181.

Bhandari, P., \& Rastogi, R. P. (1983). Chemical constituents of Trichosanthes palmata. Indian Journal of Chemistry, 22B(3), 252-256.

Calliet, S., Lorenzo, G., Cote, J., Sylvain, J.-F., \& Lacroix, M. (2013). Free RadicalScavenging Properties and Antioxidant Activity of Fractions from Cranberry Products. Food and Nutrition Sciences, 3, 337-347.

Choi, H.-K., Choi, Y. H., Verberne, M., Lefeber, A. W. M., Erkelens, C., \& Verpoorte, R. (2004). Metabolic fingerprinting of wild type and transgenic tobacco plants by ${ }^{1} \mathrm{H}$ NMR and multivariate analysis technique. Phytochemistry, 65, 857-864.

Chung, S., \& Champagne, E. (2011). Ferulic acid enhances IgE binding to peanut allergens in Western blots. Food Chemistry, 124, 1639-1642.

Crawley, M. J. (2005). Statistics: An introduction using R: Chichester: John Wiley \& Sons.

Dastmalchi, K., Cai, Q., Zhou, K., Huang, W., Serra, O., \& Stark, R. E. (2014). Solving the Jigsaw puzzle of wound-healing potato cultivars: Metabolite profiling and antioxidant activity of polar extracts. Journal of Agricultural and Food Chemistry, 62, 7963-7975.

Dastmalchi, K., Kallash, L., Wang, I., Phan, V. C., Huang, W., Serra, O., \& Stark, R. E. (2015). Defensive armor of potato tubers: Nonpolar metabolite profiling, antioxidant assessment, and solid-state NMR compositional analysis of suberinenriched wound-healing tissues. Journal of Agricultural and Food Chemistry, 63, 6810-6822.

El-Sayed, A.-A., \& Rabaliski, I. (2013). Antioxidant properties of high-lutein grain-based functional foods in comparison with ferulic acid and lutein. American Journal of Biomedical Sciences, 5, 109-125.

Farah, A., \& Donangelo, C. (2006). Phenolic compounds in coffee. Brazillian Journal of Plant Physiology, 18, 23-26.

Friedman, M. (1997). Chemistry, biochemistry and dietary role of potato polyphenols, A review. Journal of Agricultural and Food Chemistry, 47, 1523-1540.

Jackson, S. L. (2014). Research Methods: A modular approach: Stamford: Cengage Learning.

Kähkönen, M. P., Hopia, A. I., Vuorela, H. J., Rauha, J.-P., Pihlaja, K., Kujala, T. S., \& Heinonen, M. (1999). Antioxidant Activity of Plant Extracts Containing Phenolic Compounds. Journal of Agricultural and Food Chemistry, 47, 3954-3962. 
Kanatt, S. R., Chander, R., Radhakrishna, P., \& Sharma, A. (2005). Potato peel extract-A natural antioxidant for retarding lipid peroxidation in radiation processed lamb meat. . Journal of Agricultural and Food Chemistry, 53, 1499-1504.

Koufaki, M., Kiziridi, C., Papazafiri, P., Vassilopoulos, A., Varro, A., Nagy, Z., Farkas, A., \& Makriyannis, A. (2006). Synthesis and biological evaluation of benzopyran analogues bearing class III antiarrhythmic pharmacophores. Bioorganic and Medicianl Chemistry, 14, 6666-6678.

Kuijpers, T. F. M., van Herk, T., Vincken, P., Janssen, R. H., Narh, D. L., van Berkel, W. J. H., \& Gruppen, H. (2014). Potato and mushroom polyphenol oxidase activities are differently modulated by natural plant extracts. Journal of Agricultural and Food Chemistry, 62, 214-221.

Liu, J.-S., Chiang, T.-H., Wang, J.-S., Lin, L.-J., Chao, W.-C., Inbaraj, B. S., Lu, J.-F., \& Chen, B.-H. (2015). Induction of p53-independent growth inhibition in lung carcinoma cell A549 by gypenosides. Journal of Cellular and Molecular Medicine, 19, 1697-1709.

Liu, Q., Fan, Z., Li, D., Li, W., \& Guo, T. (2010). Facile Synthesis of Several OleananeType Triterpenoid Saponins. Journal of Carbohydrate Chemistry, 29, 386-402.

Madiwale, G. P., Reddivari, L., Holm, D. G., \& Vanamala, J. (2011). Storage elevates phenolic content and antioxidant activity but suppresses antiproliferative and proapoptotic properties of colored-flesh potatoes against human colon cancer cell lines. Journal of Agricultural and Food Chemistry, 59, 8155-8166.

Madiwale, G. P., Reddivari, L., Holm, D. G., \& Vanamala, J. (2012). Combined effects of storage and processing on the bioactive compounds and pro-apoptotic properties of color-fleshed potatoes in human colon cancer cells. Journal of Agricultural and Food Chemistry, 60, 11088-11096.

Magalhaes, L. M., Segundo, M. A., Reis, S., \& Lima, J. L. F. C. (2008). Methodological aspects about in vitro evaluation of antioxidant properties. Analytica Chimica Acta, 613, 1-19.

Maqsood, S., Benjakul, S., \& Shahidi, F. (2013). Emerging role of phenolic compounds as natural food additives in fish and fish products. Critical Reviews in Food Science and Nutrition, 53, 162-179.

Matsuda, H., Wang, T., Managi, H., \& Yoshikawa, M. (2003). Structural requirements of flavonoids for inhibition of protein glycation and radical scavenging activities. Bioorganic and Medicinal Chemistry, 11, 5317-5323.

Munehisa, A., Akio, F., Manabu, S., Hideki, F., Toshimitsu, H., Mineo, S., \& Naokata, M. (1986). Three new lanostanoids from Ganoderma lucidum. Journal of Natural Products, 49(4), 621-625.

Murase, T., Yokoi, Y., Misawa, K., Ominami, H., Suzuki, Y., Shibuya, Y., \& Hase, T. (2012). Coffee polyphenols modulate whole-body substrate oxidation and suppress postrandial hyperglycemia, hyperinsulinaemia and hyperlipidaemia. British Journal of Nutrition, 107, 1757-1765.

Narvaez-Cuenca, C.-E., Vincken, J.-P., Zheng, C., \& Gruppen, H. (2012). Identification and quantification of (dihydro) hydroxycinnamic acids and their conjugates in

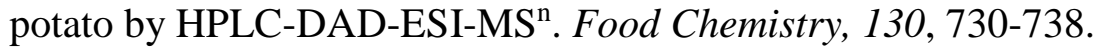


Nenadis, N., Wang, L.-F., Tsimidou, M., \& Zhang, H.-Y. (2004). Estimation of scavenging activity of phenolic compounds using the ABTS $^{\cdot+}$ assay. Journal of Agricultural and Food Chemistry, 52, 4669-4674.

Neubauer, J. D., Lulai, E. C., Thompson, A. L., Suttle, J. C., \& Bolton, M. D. (2012). Wounding coordinately induces cell wall protein, cell cycle and pectin methyl esterase genes involved in tuber closing layer and wound periderm development. Journal of Plant Physiology, 169, 586-595.

Nychas, G.-J. E., \& Tassou, C. C. (1999). Traditional preservatives- Oils and spices. In Encyclopedia of Food Microbiology, vol. 3 (pp. 1717-1722).

Re, R., Pellegrini, N., Proteggente, A., Pannala, A., Yang, M., \& Rice-Evans, C. (1999). Antioxidant activity applying an improved ABTS radical cation decolorization assay. Free Radical Biology and Medicine, 26, 1231-1237.

Reyes, L. F., \& Cisneros-Zevallos, L. (2003). Wounding stress increases the phenolic content and antioxidant capacity of purple-flesh potatoes (Solanum tuberosum L.). Journal of Agricultural and Food Chemistry, 51, 5296-5300.

Reyes, L. F., Villarrel, J. E., \& Cisneros-Zevallos, L. (2007). The increase in antioxidant capacity after wounding depends on the type of fruit or vegetable tissue. Food Chemistry, 101, 1254-1262.

Saini, P., Gayen, P., Nayak, A., \& Deepak, K. (2012). Effect of ferulic acid from Hibiscus mutabilis on filarial parasite Setaria cervi: Molecular and biochemical approaches. Parasitology International, 61(4), 10.

Schieber, A., \& Saldana, M. D. A. (2008). Potato peels: A source of nutritionally and pharmacologically interesting compounds-A review. Food, 3, 23-29.

Su, J., Wang, H., Ma, C., Liu, C., Rahman, M. R. T., Gao, C., \& Nie, R. (2016). Hypolipidemic mechanisms of gypenosides via inhibition of pancreatic lipase and reduction in cholesterol micellar solubility. European Food Research and Technology, 242, 305-312.

Thompson, M. D., Thompson, H. J., McGinley, J. N., Neil, E. S., Rush, D. K., Holm, D. G., \& Stushnoff, C. (2009). Functional food characteristics of potato cultivars (Solanum tuberosum L.): Phytochemical composition and inhibition of 1-methyl1-nitrosourea induced breast cancer in rats. Journal of Food Composition and Analysis, 22, 571-576.

Velikoca, V. B., Edreva, A. M., Tsonev, T. D., \& Jones, H. G. (2007). Singlet oxygen quenching by phenylamides and their parent compounds. Zeitschrift fuer Naturforschung, C; Journal of Biosciences, 62, 833-838.

Whitaker, E. T., \& Robinson, G. (1967). The trapazoidal and parabolic rules. In The calculus of observations. A treatise of numerical mathematics. 4th ed., (pp. 156158): Dover.

Wu, S.-B., Meyer, R. S., Whitaker, B. D., Litt, A., \& Kennelly, E. J. (2013). A new liquid chromatography-mass spectrometry-based strategy to integrate chemistry, morphology, and evolution of eggplant (Solanum) species. Journal of Chroamtography A, 1314, 154-172.

Yang, S.-X., Sun, Q.-Y., Yang, F.-M., Hu, G.-W., Luo, J.-F., Wang, Y.-H., \& Long, C.-L. (2013). Sarmentosumols A to F, new mono- and dimeric alkenylphenols from Piper sarmentosum. Planta Medica, 79(8), 693-696. 
Yang, W.-L., \& Bernards, M. A. (2007). Metabolite profiling of potato (Solanum tuberosum L.) tubers during wound-induced suberizaiton. Metabolomics, 3(2), 147-159.

Yogendra, K. N., Pushpa, D., Mosa, K. A., Kushalappa, A. C., Murphy, A., \& Mosquera, T. (2014). Quantitative resistance in potato leaves to late blight assosiated with induced hydroxycinnamic acid amides. Functional \& Integrative Genomics, 14, 285-298.

Yoshikawa, M., Arihara, S., Matsuura, K., \& Miyaset, T. (1992). Dammarane saponins from Gymnema sylvestre. Phytochemistry, 31, 237-241.

Zhang, J., Kurita, M., Shinozaki, T., Ukiya, M., Yasukawa, K., Shimizu, N., Tokuda, H., Masters, E. T., Akihisa, M., \& Akihisa, T. (2014). Triterpene glycosides and other polar constituents of shea (Vitellaria paradoxa) kernels and their bioactivities. Phytochemistry, 108, 157-170. 
Fig. 1

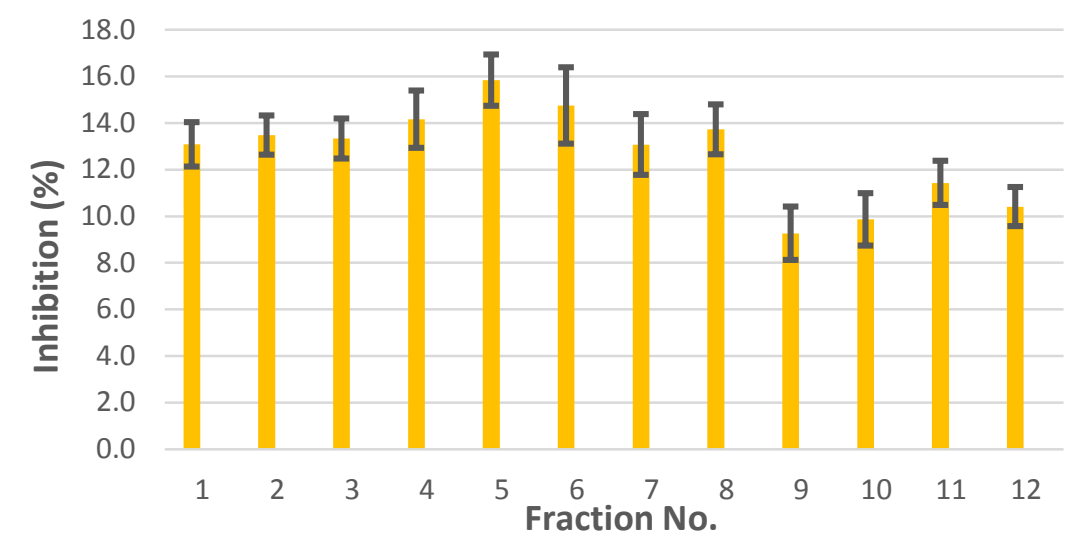

Fig. 1, top.

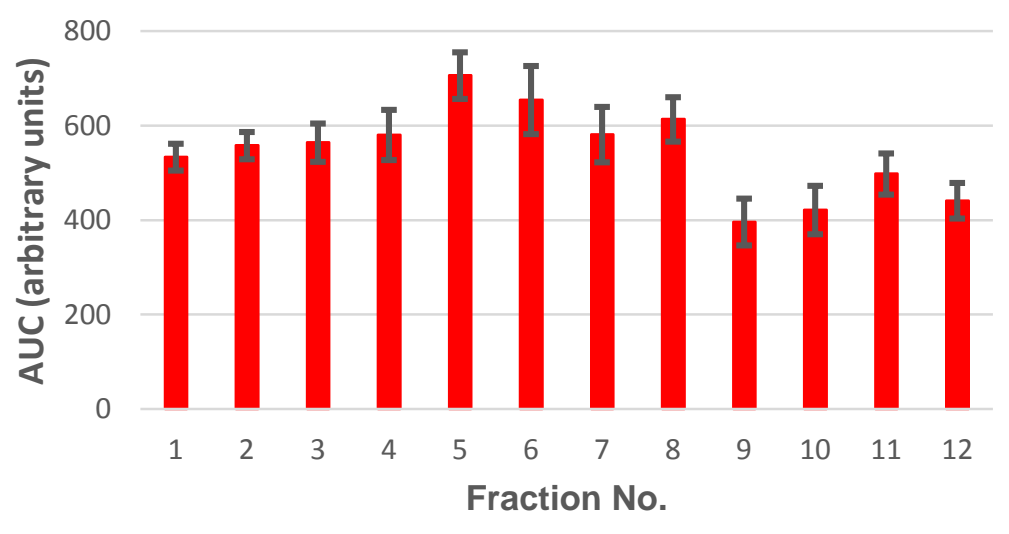

Fig. 1, middle.

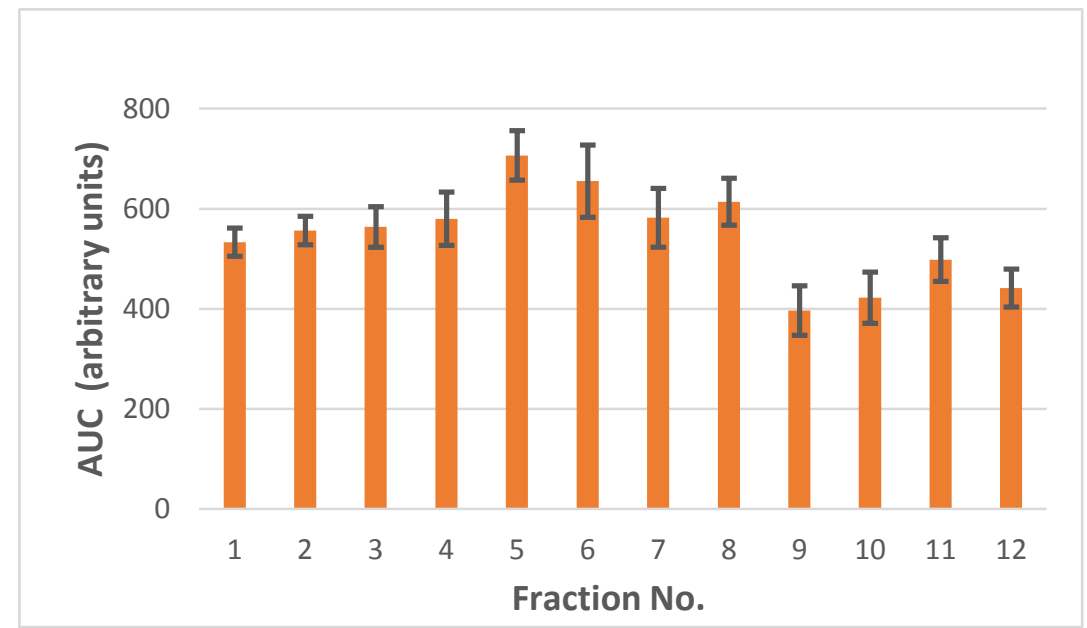

Fig. 1, bottom. 

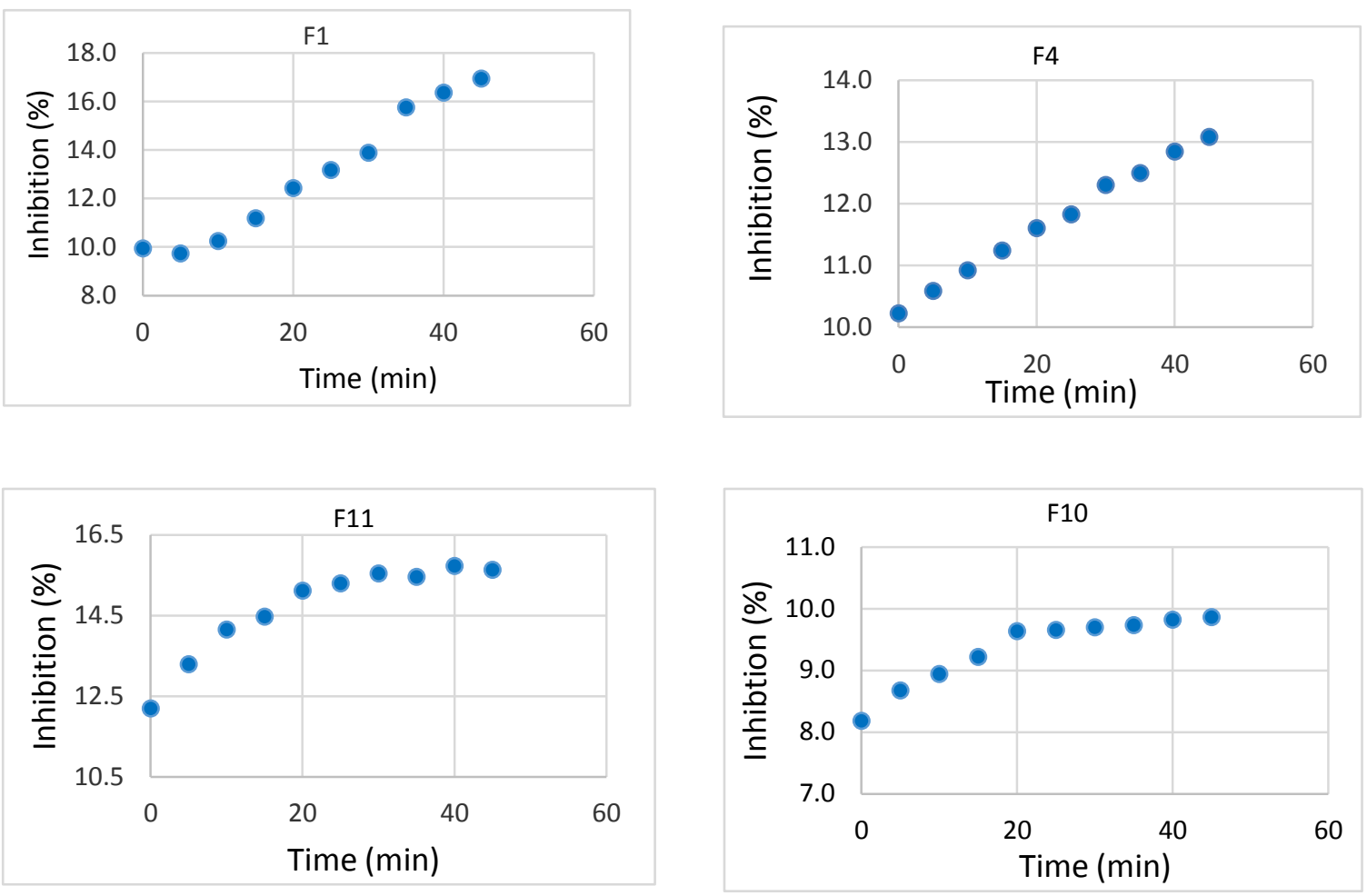

Fig. 2. 